\title{
A call for feedback on postoperative care: Do we need an automated tool?
}

\author{
Sumitra G. Bakshi, MD
}

Received: 20 September 2018/Revised: 19 October 2018/Accepted: 19 October 2018/Published online: 19 November 2018

(C) Canadian Anesthesiologists' Society 2018

\section{To the Editor,}

In the article "Learning beyond the operating room: a call for feedback on postoperative care", an important consequence of workflow in the perioperative period has been raised. ${ }^{1}$ Anesthesiology residents disagreed that they knew about the postoperative course of their patients once in the postanesthesia care unit (PACU). ${ }^{1}$ The authors correctly raise the concern that this lack of feedback from the PACU may lead to a gap in resident's training.

The role of the anesthesiologist has significantly extended beyond the intraoperative room and now encompasses preoperative clinics, the PACU, high dependency units, and pain services. There is a paucity of literature describing the actual content or implementation of a curriculum that ensures anesthesiologists have the skills necessary for extended perioperative care. ${ }^{2}$ Incidentally, many medical schools have incorporated a longitudinal clerkship for training medical students on anesthesia rotations, and are essentially patient centric; that is, following the patient in the preoperative, intraoperative, and postoperative phases. ${ }^{2}$ This longitudinal structure helps the learner understand the outcome and benefit of each intervention while strengthening the rapport with the patient. ${ }^{3}$

Postoperative visits by the anesthesiologist have been critically reviewed. ${ }^{4}$ First hand feedback from the patient

This letter is accompanied by a reply. Please see Can J Anesth 2018; 65: this issue.

\section{S. G. Bakshi, MD ( ()}

Department of Anesthesiology, Critical Care and Pain, Tata Memorial Hospital, Homi Bhabha National Institute, Mumbai, India

e-mail: sumitrabakshi@yahoo.in helps the resident understand the patient's anesthesia experience and pain relief. ${ }^{4}$ These visits can also help design institute-specific protocols. ${ }^{4}$ Should this clinical follow-up be replaced with automated tools, which are prone to failures and limited to the type of data captured? In person, follow-up and feedback provides human interaction between care givers and patients and results in greater patient satisfaction. ${ }^{5}$ One wonders if rather than turning to an automated system, we should instead design a curriculum and work pattern that permits and insists on direct patient feedback.

Conflicts of interest None declared.

Editorial responsibility This submission was handled by Dr. Gregory L. Bryson, Deputy Editor-in-Chief, Canadian Journal of Anesthesia.

Funding Departmental funding only.

\section{References}

1. Khan N, Mueller A, Mitchell JD, Bose S. Learning beyond the operating room: a call for feedback on postoperative care. Can J Anesth 2018; 65: 1080-1.

2. Alem N, Cohen N, Cannesson M, Kain Z. Transforming perioperative care: the case for a novel curriculum for anesthesiology resident training. A A Case Rep 2016; 6: 373-9.

3. Sullivan KR, Rollins MD. Innovations in anaesthesia medical student clerkships. Best Pract Res Clin Anaesthesiol 2012; 26: 2332.

4. Bajwa SJ, Takrouri MS. Post-operative anesthesia rounds: need of the hour. Anesth Essays Res 2013; 7: 291-3.

5. Rentmeester $C$. Heeding humanity in an age of electronic health records: Heidegger, Levinas, and Healthcare. Nurs Philos 2018; 19: e12214. 